\title{
Coulomb gap in one-dimensional disordered electronic systems
}

\author{
Amit Dutta, ${ }^{1}$ Lars Fritz, ${ }^{2}$ and Diptiman Sen $^{3}$ \\ ${ }^{1}$ Department of Physics, Indian Institute of Technology, Kanpur 208016, India \\ ${ }^{2}$ Institut für Theoretische Physik und Astrophysik, Universität Würzburg, Am Hubland, 97074 Würzburg, Germany \\ ${ }^{3}$ Centre for Theoretical Studies, Indian Institute of Science, Bangalore 560012, India
}

\begin{abstract}
We study a one-dimensional system of spinless electrons in the presence of a long-range Coulomb interaction (LRCI) and a random chemical potential at each site. We present a Tomonaga-Luttinger liquid description of the system. We use the bosonization technique followed by the replica trick to average over the quenched randomness. An expression for the localization length of the system is obtained using the renormalizationgroup method and also a physical argument. We then find the density of states for different values of the energy; we get different expressions depending on whether the scale of energy is larger than or smaller than the inverse of the localization length. We work in the limit of weak disorder where the localization length is very large, at that length scale, the LRCI has the effect of reducing the interaction parameter $K$ to a value much smaller than the noninteracting value of unity.
\end{abstract}

In a strongly localized electron system with a long-range Coulomb interaction (LRCI) between the electrons, the density of states (DOS) is expected to exhibit a gap at the Fermi energy called the "Coulomb gap." 1 A phenomenological argument ${ }^{1}$ suggests that the DOS should go as $D(\omega)$ $\sim|\omega|^{d-1}$, where $d$ is the spatial dimensionality of the system and $\omega$ is measured from the Fermi energy. We shall consider here the one-dimensional case where the DOS is expected to show a logarithmic behavior in the extremely localized limit. $^{2-5}$ In low-dimensional systems, the electron-electron interactions play a dominant role leading to a behavior significantly different from that of conventional Fermi liquids. Short-range repulsive interactions between the electrons give rise to a Tomonaga-Luttinger liquid (TLL), ${ }^{6-8}$ whereas the LRCI is believed to lead to a Wigner crystal. ${ }^{9}$

It is well known that for a one-dimensional noninteracting electron system with random disorder, all the states are localized due to repeated backscatterings of the electrons. ${ }^{10}$ However, the DOS of noninteracting electrons is finite at the Fermi energy. ${ }^{11}$ The presence of the LRCI together with random impurities modifies the DOS in a drastic way and supposedly leads to the Coulomb gap behavior. In an earlier study, Vojta and $\mathrm{John}^{5}$ studied a one-dimensional electron system, with LRCI and randomness, in the extremely localized limit where the overlap of electronic wave functions can be neglected. They find the form of the DOS to be

$$
D(\omega) \sim\left(\ln \frac{E_{c}}{|\omega|}\right)^{-1},
$$

where $E_{c}$ is a cutoff energy. ${ }^{3,4}$ In a recent paper, Lee ${ }^{12}$ derived the expression of the DOS of a one-dimensional system of spinless electrons with LRCI and impurities at random positions in the opposite limit where the quantum effects (electron hopping) play a dominant role, and the pinning is weak, i.e., the localization length is much larger than the interimpurity distance. Following studies of a pinned Wigner crystal $^{13,14}$ and using a semiclassical approximation, Lee ${ }^{12}$ finds the DOS at low energy to be

$$
D(\omega) \sim|\omega|^{\sqrt{1+\eta} / 2},
$$

where the exponent $\eta$ [defined in Eq. (15) below] is determined by the localization length of the system.

In this paper, we study a model of spinless electrons with LRCI as in Ref. 12 (with quantum effects, i.e., with the electron hopping term) in the presence of a random chemical potential at each site. We find that a TLL picture, ${ }^{8}$ rather than a semiclassical approach, ${ }^{12}$ provides a convenient description of the system. The localization length $L_{0}$ is derived using a renormalization group ( $R G)$ study of the effective disorderaveraged bosonized action, ${ }^{15}$ and is found to be very large in the limit of weak disorder. (We also present a simple physical understanding of the expression for $L_{0}$.) Due to the LRCI, the interaction parameter $K$ of the TLL is found to be effectively a function of the length scale. At the length scale $L_{0}$, the value of $K$ is given by a value much smaller than unity (which is the value of $K$ for the noninteracting system). We should remark here that the present one-dimensional disordered electron system with LRCI appears to be a unique example of a TLL with $K \ll 1$.

Once we make use of a TLL description, the DOS can be found in a standard way. ${ }^{8,16}$ We will show that for $\omega$ $\gtrsim v_{F} / L_{0}$, the DOS is given by

$$
\begin{gathered}
D(\omega) \sim|\omega|^{\beta}, \\
\beta=\frac{\sqrt{1+\eta}}{2}+\frac{1}{2 \sqrt{1+\eta}}-1,
\end{gathered}
$$

where, unlike a standard TLL, $\eta$ itself depends on $\omega$ as discussed below. [Equation (3) resembles the result of Lee ${ }^{12}$ for $\eta \gg 1$; however, $\eta$ is not taken to be a function of $\omega$ in Ref. 12.] For $\omega \lesssim v_{F} / L_{0}$, we will argue that the DOS is given by the expression in Eq. (1).

The Hamiltonian of a disordered system of spinless electrons with LRCI consists of three parts, $H=H_{0}+H_{C}$ 
$+H_{\text {random }}$. The noninteracting part $H_{0}$ and the Coulomb interaction part $H_{C}$ are written in terms of the continuum electron fields as follows,

$$
\begin{gathered}
H_{0}=v_{F} \int_{-\infty}^{\infty} d x\left(-i \psi_{R}^{\dagger} \partial_{x} \psi_{R}+i \psi_{L}^{\dagger} \partial_{x} \psi_{L}\right), \\
H_{C}=\frac{1}{2} \int_{-\infty}^{\infty} d x d y U(x-y) \rho(x) \rho(y),
\end{gathered}
$$

where the form of $U(x-y)$ will be specified later, and $\rho$ is the electron density. [The fields $\psi_{R}\left(\psi_{L}\right)$ are the right-moving (left-moving) electron operators.] The random part of the Hamiltonian consists of two parts, (i) the forward-scattering part $H_{f}$, where the scattered electrons remain in the vicinity of the same Fermi point, and (ii) the backward-scattering part $H_{b}$, where an electron is scattered from $-k_{F}$ to $k_{F}$, or vice versa.

We now rewrite the full Hamiltonian in the language of bosonization. ${ }^{8,16}$ The low-energy and long-wavelength excitations of the noninteracting part can be written as

$$
H_{0}=\frac{v_{F}}{2 \pi} \int_{-\infty}^{\infty} d x\left\{\left[\partial_{x} \theta(x)\right]^{2}+\left[\partial_{x} \phi(x)\right]^{2}\right\},
$$

while the Coulomb part is given by

$$
H_{C}=\frac{1}{2 \pi^{2}} \int_{-\infty}^{\infty} d x d y \quad U(x-y) \partial_{x} \phi(x) \partial_{y} \phi(y) .
$$

The forward-scattering part is given by

$$
\begin{aligned}
H_{f} & =\int_{-\infty}^{\infty} d x h(x)\left[\psi_{R}^{\dagger}(x) \psi_{R}(x)+\psi_{L}^{\dagger}(x) \psi_{L}(x)\right] \\
& =-\frac{1}{\pi} \int_{-\infty}^{\infty} d x h(x) \partial_{x} \phi(x) .
\end{aligned}
$$

The forward scattering is due to the real random field $h(x)$; its effect can be taken care of by a rescaling of the bosonic field $\phi,{ }^{15}$ and we shall henceforth ignore this part. The backscattering part can be written as

$$
\begin{aligned}
H_{b} & =\int_{-\infty}^{\infty} d x\left[\xi(x) \psi_{R}^{\dagger}(x) \psi_{L}(x)+\xi^{*}(x) \psi_{L}^{\dagger}(x) \psi_{R}(x)\right] \\
& =\frac{1}{\pi \alpha} \int_{-\infty}^{\infty} d x\left[\xi(x) e^{i\left(2 \phi(x)+2 k_{F} x\right)}+\text { H.c. }\right]
\end{aligned}
$$

where H.c. denotes the Hermitian conjugate. The backscattering is due to a complex random field $\xi(x)$ with the probability distribution

$$
P[\xi(x)]=\exp \left[-D_{\xi}^{-1} \int d x \xi^{*}(x) \xi(x)\right],
$$

so that $\langle\xi(x)\rangle=0$ and $\left\langle\xi^{*}(x) \xi\left(x^{\prime}\right)\right\rangle=D_{\xi} \delta\left(x-x^{\prime}\right)$.

The Hamiltonians given in Eqs. (6), (7), and (9) constitute the complete low-energy description of the model in the bosonic language. We now write the Coulomb interaction in Eq. (7) in momentum space as

$$
\begin{array}{r}
\frac{1}{2 \pi^{2}} \int d x d y U(x-y) \partial_{x} \phi(x) \partial_{y} \phi(y) \\
\quad=\frac{1}{4 \pi^{3}} \int d k k^{2} \hat{U}(k) \hat{\phi}(k) \hat{\phi}(-k),
\end{array}
$$

where $\hat{U}(k)$ is the Fourier transform of the Coulomb interaction. We can expand $\hat{U}(k)$ in powers of $k$ as

$$
\hat{U}(k)=\hat{U}_{0}+\hat{U}_{1} k+\hat{U}_{2} k^{2}+\cdots .
$$

Considering the scaling dimensions of the various terms in Eq. (12), we conclude that the constant term is marginal under renormalization, whereas the terms involving powers of $k$ are irrelevant. Hence, the important contribution arising from the Coulomb interaction in Eq. (11) can be written as

$$
H_{C}=\frac{\hat{U}_{0}}{4 \pi^{3}} \int d k k^{2} \hat{\phi}(k) \hat{\phi}(-k)=\frac{\hat{U}_{0}}{2 \pi^{2}} \int d x\left[\partial_{x} \phi(x)\right]^{2} \text {. }
$$

The constant $\hat{U}_{0}$ is given by

$$
\hat{U}_{0}=\int_{-L / 2}^{L / 2} d x \frac{q^{2}}{\sqrt{x^{2}+d^{2}}}=2 q^{2} \ln \frac{L}{d},
$$

where $q$ is the charge of the electron, and $d$ is the width of the wire which is of the order of the lattice spacing $a$. The meaning of the cutoff length $L$ will be discussed below. (We have assumed that $L \gg a, d$, this will be justified later.) We now define a dimensionless quantity

$$
\eta=\frac{2 q^{2}}{\pi v_{F}} \ln \frac{L}{a} .
$$

Then the Hamiltonian can be written in the form $H=H_{0}$ $+H_{C}+H_{b}$, where

$$
H_{0}+H_{C}=\frac{v_{F}}{2 \pi} \int d x\left[\left(\partial_{x} \theta\right)^{2}+(1+\eta)\left(\partial_{x} \phi\right)^{2}\right] .
$$

Equation (16) can be brought into a standard form by defining

$$
K=\frac{1}{\sqrt{1+\eta}} \quad \text { and } \quad u=v_{F} \sqrt{1+\eta}
$$

We then arrive at the expression

$$
H_{0}+H_{C}=\frac{u}{2 \pi} \int d x\left[K\left(\partial_{x} \theta\right)^{2}+\frac{1}{K}\left(\partial_{x} \phi\right)^{2}\right] .
$$

In the imaginary time representation, we obtain

$$
S_{0}+S_{C}=\frac{u}{2 \pi} \int d x d \tau\left[K\left[\partial_{\tau} \phi(x, \tau)\right]^{2}+\frac{1}{K}\left[\partial_{x} \phi(x, \tau)\right]^{2}\right] \text {, }
$$




$$
S_{b}=\frac{1}{\pi \alpha} \int d x d \tau\left[\xi(x) e^{i\left[2 \phi(x, \tau)+2 k_{F} x\right]}+\text { H.c. }\right] .
$$

The randomness is dealt with by using the standard replica method and averaging over the randomness using the distribution in Eq. (10). The final form of the disorder-averaged $n$-replicated action is found to be ${ }^{15}$

$$
\begin{aligned}
S_{n}= & \frac{u}{2 \pi} \sum_{a} \int d x d \tau\left[K\left[\partial_{\tau} \phi^{a}(x, \tau)\right]^{2}+\frac{1}{K}\left[\partial_{x} \phi^{a}(x, \tau)\right]^{2}\right] \\
& -\frac{D_{\xi}}{(\pi \alpha)^{2}} \sum_{a, b} \int d x d \tau d \tau^{\prime} \cos \left[2 \phi^{a}(x, \tau)-2 \phi^{b}\left(x, \tau^{\prime}\right)\right],
\end{aligned}
$$

where $a, b$ are the replica indices running from 1 to $n$. The RG equations of $D_{\xi}, K$, and $u$ are given by ${ }^{15}$

$$
\begin{gathered}
\frac{d D}{d l}=(3-2 K) D, \\
\frac{d K}{d l}=-\frac{1}{2} K^{2} D, \quad \text { and } \quad \frac{d u}{d l}=-\frac{u K}{2} D,
\end{gathered}
$$

where $D=\left(2 D_{\xi} \alpha\right) / \pi u^{2}$ and $l=\ln (L / a)$ is the logarithm of the length scale. For $K>3 / 2$, the disorder is irrelevant. For $K<3 / 2$, the disorder grows under RG and leads to localization. The localization length, denoted by $L_{0}$, can be obtained by integrating the RG equations in Eq. (21). At the length scale $a$, we begin with some values of $D$ (much smaller than 1 ), and $u, K$ given in Eq. (17). [The latter requires a knowledge of the length scale $L$ through Eq. (15), this will be determined self-consistently by the solution of Eqs. (21).] Since we are assuming that $K \ll 1$, the quantities $K$ and $u$ flow very little while $D$ flows from a small number of order $D_{\xi}$ to a number of order 1 . The length scale at which the disorder strength becomes of order 1 is given by

$$
\frac{L_{0}}{a} \sim\left(\frac{1}{D_{\xi}}\right)^{1 /(3-2 K)} .
$$

The physical meaning of $L_{0}$ will be discussed below.

In the limit of weak disorder $\left(D_{\xi} \rightarrow 0\right)$, the form of the localization length in Eq. (22) implies, in a self-consistent manner, that (i) $L_{0} \gg a$, (ii) the parameter $\eta \gg 1$ [replacing $L$ by $L_{0}$ in Eq. (15)], and (iii) the interaction parameter $K \ll 1$ due to Eq. (17). Hence the localization length in the $K \rightarrow 0$ limit assumes the classical value $L_{0} / a \sim\left(1 / D_{\xi}\right)^{1 / 3}$.

The significance of the localization length is as follows. Although two electrons which are separated by more than the distance $L_{0}$ do interact with each other through the Coulomb potential, the overlap of their wave functions is exponentially small, and hence their positions are uncorrelated. Such interactions will therefore only contribute to a uniform and static chemical potential which is the same for all electrons. Namely, the Coulomb potential felt by an electron from other electrons which are separated from it by a distance larger than $L_{0}$ is described by a part of Eq. (5) given by

$$
\begin{aligned}
H_{C}= & \frac{1}{2} \int_{-\infty}^{\infty} d x \rho(x) \rho_{0}\left[\int_{x+L_{0}}^{\infty} d y U(x-y)\right. \\
& \left.+\int_{-\infty}^{x-L_{0}} d y U(x-y)\right],
\end{aligned}
$$

where $\rho_{0}$ is the average density. Equation (23) represents a uniform one-body potential. On the other hand, when two electrons are closer to each other than $L_{0}$ that their positions are correlated, then their Coulomb interaction has to be included in the quadratic part of the bosonic Hamiltonian [Eq. (7) to be specific] which governs the density fluctuations. In short, portions of the system which are separated by distances larger than $L_{0}$ are uncorrelated, whereas, within a distance $L_{0}$, the system can be described in terms of a TLL with $K \ll 1$.

Before proceeding further, we would like to present a simple physical understanding of the important result in Eq. (22) which does not make use of the replica idea. First, let us consider a weak $\delta$-function impurity of strength $V_{0}$ at one point in a TLL. According to a RG equation derived by Kane and Fisher, ${ }^{17}$ the impurity strength $V$ flows according to the equation $d V / d l=(1-K) V$; hence, at a distance scale $L$, we have $V(L) \sim V_{0}(L / a)^{1-K}$. From quantum mechanics, we know that the reflection amplitude for an electron scattering from a single impurity is proportional to $V$ if $V / v_{F}$ is small. Now suppose that each site in the lattice has a random impurity of strength $V(x)$ which satisfies $\langle V(x) V(y)\rangle$ $=D_{\xi} \delta(x-y)$. This means that at each site, $V(x)$ is of the order of $\sqrt{D_{\xi}}$, and its sign is random. Over a length $L$, there are $L / a$ reflections since there is a reflection at each site. In order that these $L / a$ reflections should add up to a total reflection amplitude of order 1 (which would localize the electron), we must have $\sqrt{L / a} V(L) \sim 1$ (assuming that $L / a$ is large, and using the well-known result for the sum of a large number of random terms in a Brownian motion). This gives us the estimate $L_{0} / a \sim 1 /\left[V\left(L_{0}\right)\right]^{2}, \quad$ i.e., $\quad L_{0} / a$ $\sim 1 /\left[V_{0}\left(L_{0} / a\right)^{1-K}\right]^{2}$. Since $V_{0} \sim \sqrt{D_{\xi}}$, we obtain $L_{0} / a$ $\sim\left(1 / D_{\xi}\right)^{1 /(3-2 K)}$.

We are now ready to discuss the density of states. For energies satisfying $\omega \gtrsim v_{F} / L_{0}$, the disorder parameter $D$ is small, and our system can be described by a clean TLL. The DOS of a TLL is given by the relation ${ }^{8}$

$$
\begin{gathered}
D(\omega) \sim|\omega|^{\beta}, \\
\beta=\frac{(1-K)^{2}}{2 K}=\frac{\sqrt{1+\eta}}{2}+\frac{1}{2 \sqrt{1+\eta}}-1,
\end{gathered}
$$

where we have used the relation $K=1 / \sqrt{1+\eta}$. This is the result quoted in Eq. (3). However, we now have to determine what value of $L$ one should take in Eq. (15) in order to determine the value of $\eta$ to be used in Eq. (24).

It turns out that $\omega$ itself determines the appropriate value of $\eta$ to use in Eq. (24). If $\omega=v_{F} / L_{0}$, the length scale of interest is $v_{F} / \omega=L_{0}$. The value of $\eta$ to use in Eq. (24) is then given self-consistently by Eqs. (15) (with $L$ replaced by $\left.L_{0}\right)$, (17), and (22). However, if $\omega>v_{F} / L_{0}$, then the relevant 
length scale is $L=v_{F} / \omega$ which is smaller than $L_{0}$. Then the Coulomb interaction should be cut off at the length scale $L$ as indicated in Eq. (14). This follows from the basic idea behind the RG method, namely, that the properties of a system at a length scale $L$ (or energy scale $v_{F} / L$ ) are governed by the modes whose wavelengths are smaller than $L$. [The modes with wavelengths larger than $L$ only contribute to a uniform and static chemical potential in the sense described in Eq. (23).] If the Coulomb interaction is cut off at the distance $L$, the value of $\eta$ to be used in Eq. (24) is simply given by Eq. (15). We thus conclude that for $\omega \gtrsim v_{F} / L_{0}$, the DOS is given by Eq. (24) where $\eta$ depends on $\omega$ through Eq. (15) with $L=v_{F} / \omega$.

We note that the expression in Eq. (3) agrees with the semiclassical result in Eq. (2) in the limit $\eta \gg 1$. Moreover, Eq. (3) correctly reproduces the expression of the DOS of a noninteracting disordered electronic system (for which $q^{2}$ $=0$, and therefore $\eta=0$ and $K=1$ ), where there is a finite DOS at the Fermi energy. ${ }^{11}$

We now consider the case of low energies satisfying $\omega$ $<v_{F} / L_{0}$. Then Eqs. (21) imply that the disorder strength flows to a value of order 1; hence the system cannot be described by a clean TLL. However, this is precisely the regime described by the localized limit discussed in Refs. 3-5, where the localization length $L_{0}$ is smaller than the length scale of interest, namely, $L=v_{F} / \omega$. We therefore expect the DOS in this regime to be described by Eq. (1).

We have thus found expressions for the DOS in the regimes $\omega \gtrsim v_{F} / L_{0}$ and $\omega \lesssim v_{F} / L_{0}$ (strictly speaking, $\omega$ $\gg v_{F} / L_{0}$ and $\left.\omega \ll v_{F} / L_{0}\right)$. The DOS must of course cross over smoothly from one regime to the other, but this cross over is difficult to determine analytically.

Let us summarize our key results. In the limit of weak disorder, the system is power-law correlated only over a large distance of order $L_{0}$, called the localization or pinning length. At that length scale, the system is described by a TLL in which the LRCI reduces the interaction parameter $K$ to a value much smaller than unity. [We note that in the absence of disorder, the LRCI leads to a Wigner $\operatorname{crystal}^{9}$ for which a TLL description is not valid. In our formalism, we can see this by noting that if the disorder was absent (i.e., $D_{\xi}=0$ ), we would have $L_{0}=\infty, \eta=\infty$, and $K=0$; hence the TLL description would no longer be valid.] Up to the length scale $L_{0}$, the TLL description enables us to derive the DOS easily using well-known relations without having to make use of a semiclassical approach. ${ }^{12}$ Beyond the length scale $L_{0}$, the TLL description is no longer valid since the disorder strength is of order 1. In this regime, the DOS is given by Eq. (1); thus as $\omega \rightarrow 0$, the DOS goes to zero logarithmically rather than as a power law.

A.D. and L.F. gratefully acknowledge important and encouraging discussions with R. Oppermann. A.D. also acknowledges the hospitality of the Institut für Theoretische Physik und Astrophysik, Universität Würzburg, where the initial part of the work was done, and Deutsche Forschunggemeinschaft for financial support via Project No. OP28/5-2. D.S. thanks the Department of Science and Technology, India for financial support through Grant No. SP/S2/ M-11/00.
${ }^{1}$ A.L. Efros and B.I. Shklovskii, J. Phys. C 8, L49 (1975).

${ }^{2}$ A.E. White, R.C. Dynes, and J.P. Garno, Phys. Rev. Lett. 56, 532 (1986).

${ }^{3}$ M.E. Raich and A.L. Efros, Pis'ma Zh. Éksp. Teor. Fiz. 45, 225 (1987) [JETP Lett. 45, 280 (1987)].

${ }^{4}$ A. Möbius and M. Richter, J. Phys. C 20, 539 (1987).

${ }^{5}$ T. Vojta and W. John, J. Phys.: Condens. Matter 5, 57 (1993).

${ }^{6}$ J. Voit, Rep. Prog. Phys. 58, 977 (1995).

${ }^{7}$ H.J. Schulz, G. Cuniberti, and P. Pieri, cond-mat/9807366 (unpublished); in Field Theories for Low-dimensional Condensed Matter Systems, edited by G. Morandi, A. Tagliacozzo, and P. Sodano (Springer, Berlin, 2000).

${ }^{8}$ S. Rao and D. Sen, cond-mat/0005492 (unpublished); in Field Theories in Condensed Matter Systems, edited by S. Rao (Hin- dustan Book Agency, New Delhi, 2001).

${ }^{9}$ H.J. Schulz, Phys. Rev. Lett. 71, 1864 (1993).

${ }^{10}$ P.A. Lee and T.V. Ramakrishnan, Rev. Mod. Phys. 57, 287 (1985).

${ }^{11}$ B. Halperin, Phys. Rev. 139, A104 (1965).

${ }^{12}$ H.C. Lee, Phys. Rev. B 66, 052202 (2002).

${ }^{13}$ H. Maurey and T. Giamarchi, Phys. Rev. B 51, 10833 (1995).

${ }^{14}$ H. Fukuyama and P.A. Lee, Phys. Rev. B 17, 535 (1978); P.A. Lee and H. Fukuyama, ibid. 17, 542 (1978).

${ }^{15}$ T. Giamarchi and H.J. Schulz, Phys. Rev. B 37, 325 (1988).

${ }^{16}$ A.O. Gogolin, A.A. Nersesyan, and A.M. Tsvelik, Bosonization and Strongly Correlated Systems (Cambridge University Press, Cambridge, 1998).

${ }^{17}$ C.L. Kane and M.P.A. Fisher, Phys. Rev. B 46, 15233 (1992). 